\title{
Organizational resilience a new paradigm for the knowledge society
}

\author{
Koninckx Guy
}

\author{
Bachelor in Sciences of work - Psychologist - Systemicien \\ Namastis - Human Resources \& Change Management \\ G.I.R.O.S. \\ guy.koninckx@skynet.be
}

\begin{abstract}
Our organizations operate in an environment of turbulence and crises. Loss of marks and sense constitutes part of everyday life for our organizations. Futhermore, it should be noted that in recent decades social security systems covering many areas have been established. Currently uncertainty becomes a certainty. How to bounce back in such circumstances? What happens to the paradigms that underlie the functioning of organizations? Which functions do they play? Which paradigms are mediated by resilience? What is the added value of resilience? Which perspectives for the future?
\end{abstract}

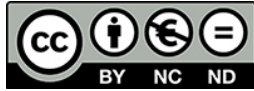

Keywoords: resilience - paradigm - crisis - knowledge

\section{The context}

In the early nineties the concept of resilience appears. While the concept falls under the physics it has been used by analogy and considered in general as" the ability to bounce back". Over the years several disciplines have appropriated the concept. Let us consider this observation as a symptom of the functioning of our organizations.

Individuals and teams evolve within organizations in a context of incessant turbulences. The upheavals that trouble an organization challenge individuals and teams. The players in the organization, the stakeholders have to solve an equation with several unknowns. The representations of their organization is not spared.

Several scenarios are possible: the withdrawal into oneself and allow the event handle the situation or activate the resilience capacities to cope and seize the opportunity to bounce back. Different postures to change are emerging. Observed behaviors are build upon different paradigms. In a chaotic context some paradigms are reinterpreted, others undermined and newone are emerging.

What about a governance based on organizational resilience ? Which paradigm is at work? We are engaged in major changes and ignore the outlines of those. Clarify the concepts of resilience and paradigm are essential to answer the question: is organizational resilience a new paradigm for the knowledge society

\section{Resilience}

Originally, "resilience" is a concept coming from the physics sciences: "the defining characteristic of the resistance to shocks of materials." Resilience is a mechanical property of an inert material that consists to keep his property after an undergone "shock" suffered. The consequences can be: stability, breaking or rupture of the material. The material needs a time (t) to regain his property. While this material undergoes the action of an external agent it retains his property within certain limits. Is resilient "what has resistance to impact." That is how comes the idea of resistance to shocks. The effort limits of plasticity and the breaking point can be identified. Many disciplines have taken over the concept of "resilience" and are using it by analogy. Without being exhaustive, let us quote: computer science, psychology, finance, ecology, metallurgy, economics, ergonomics, social sciences, management etc ... 
Boris Cyrulnik one of the first working on the topic in France, defines resilience as "the capacity to succeed in living and to grow in an acceptable way in spite of stress or adversity which normally entails a serious risk of a fatal outcome". Vanistendal's definition adds another dimension. He highlights the importance of interactions between the individual and the environment that surrounds him. He passes from an isolated individual to the notion of group and community. "Resilience is the capacity of a person or a group to be projected in the future in spite of destabilizing events, difficult living conditions, sometimes severe traumas".

For C Aschan-Leygonie "Resilience is equivalent to the notion of stability of a system around an equilibrium point. After a disturbance, the system reacts to contrary often positive, creatively, through multiple changes and adjustments. In this sense, resilience is the property of a system that still retains the same path after a disturbance. The system integrates by evolving transformations. In this perspective, change, and disruption that triggers it are not necessary trauma. »(1)

The limits of the paradigm, based on the equilibrium for open systems appear. The idea that there are several possible situations implies the possibility for a system to be located far from equilibrium without collapsing.

Turbulences can be experienced on the mode of threat or opportunity. Experienced as a threat they carry perspectives for deconstruction; while experienced as an opportunity they open the perspective for rebuilding or creation. The issue is in the field of management of destabilizing event.

Organizations can address turbulence and shocks in different ways depending on the context and events.

The challenge lies in terms of management the destabilizing event. In addition to the avoidance process favoring stability or return to equilibrium, there are other more appropriate process for moving towards new equilibria or towards innovation.

Many elements of resilience identified at individual level can be transposed to the organization and some organizational factors are favorable to the emergence of resilience process.

In the "General systems theory", Ludwig von Bertalanffy studied open systems "in balance", that is to say systems which are subject to a set of changes that lead to the same stable state for given conditions.

In human systems, we distinguish the negative, positive and evolutionary feedback, depending on their role in relation to maintaining a known balance or searching for another balance. (2)

For systemics resistance and reconstruction are similar to regulation mechanisms we find in the homeostasis process. It is to bring back the whole to its initial state, to ensure its survival or to regain balance. Customs forwarded particularly through education and the rules will play a key role here. The mention or reference to the construction of a completely different nature (change from another logic level) is rather the result of a bifurcation and is similar to a mutation. Indeed, it could be that the shock, internal or external, is an opportunity to see the emergence of a new order, a total transformation in a way a rupture.

\section{The paradigm}

In a situation of confrontation, instability and turbulence, reflections relating to "sense" emerge. The lack of sense, loss of sense or nonsense are also at the heart of debates. It is like events challenge simultaneously two levels. On the one hand the organizational level of its purpose, vision and values. On the other hand the level of individuals in their work and life outside.

What are the keys to the sensemaking for the ones and for others? The way of thinking is based on a well-defined base, which can be regarded as a model of thought close beliefs. This model, the representation of the world, is a paradigm. The set of assumptions that determine how to see things, to represent them. These assumptions influence the way we perceive the world and react to this 
perception. It is the basis of an explanation system and serves as a guide to action regardless of the areas.

To define the concept of paradigm, we will retain the definition of B. Vidaillet "The paradigm is the set of assumptions, usually implicit, the elements that constitute the world, their actions, their relationships and how we can know them. His influence will be even stronger than it is shared by the members of the organization and is well articulated."(3)

All these assumptions and values influence the way of perceiving reality and react to this perception. This mode of representation allows the individual to define what surrounds, to understand it or to foresee it. This way of considering things is in some way an analysis grid.

In a collective sense, the paradigm is a widely accepted system of representation in a particular area by a group. By extension, paradigms may vary depending on the group and change over time. The new paradigm interact with the vision. It's a new system of explanation and can lead to new strategies for action. A paradigm shift necessarily means an upheaval because it directly affects the vision of the reality and the explanation be given regarding this reality. Even though they are often to an implicit level and are not fixed for eternity. These "scripts" play a major role. It may be useful to understand the way of their development.

\subsection{The development of a paradigm}

Concretely, the experience of the individual is punctuated by diverse experiences and situations that lead this one to think, to interpret and to reach a provisional finding. The redundancy of this procedure leads to consolidate its conclusion. It is as if the functional analysis is complete, the programmer is able to write the program. It is at this point that the paradigm is build. It serves as as a guide at the behavioral and the feelings level. Having paradigms saves this process for similar situations. It works as a collective unconscious level of an organization. These paradigms are ignored by others, the individual can access it through observations of the organization, operations and redundancies. This representation can be seen through, for others, as a simplification of reality.

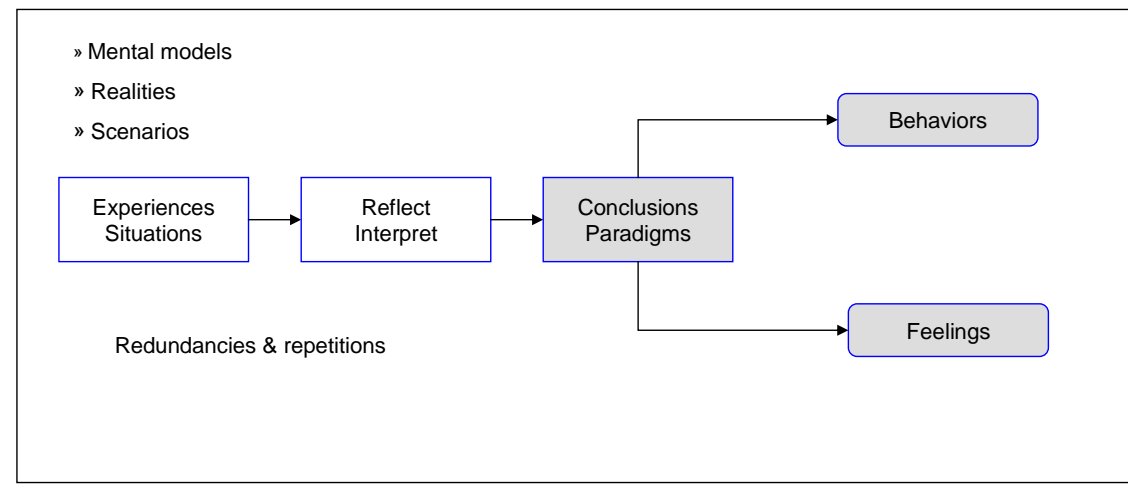

The development of a paradigm

\subsection{What is the function of the paradigm}

All these paradigms feeds beliefs, mental models and organizational identity previously developed. Individuals and organizations need to rely on a sufficient number of permanences. Beliefs and paradigms structure. They avoid to evolve in permanent doubts. They respond to a need for security and influence which we can qualify as "normative". Clearly it must be recognized the paradigms exercise a function. This does not exclude that, while relevant, a paradigm becomes inadequate. Why? Because it may hide in some moments, misunderstandings, errors, and provide 
simplifications and false certainties. Furthermore, beliefs are not immune to some mode effects. A rereading of the function of paradigm illustrates the interest in outlining the system with a view to redecision and change.

\section{Paradigms conveyed by resilience.}

When the paradigm is relevant decisions are becoming a sort of "action's intelligence". Otherwise, the logical thought and action produce "malfunctions". Every day the news informs us about the future of groups fixed on a paradigm rather than take the risk to innovate. When a paradigm becomes obsolete, it usually results in a crisis and transition periods. The crisis can be understood then as a signifying sign. We can put forward the hypothesis it addresses the relationship maintained by the decision makers of a organization or a country to the reality. It is a time to break with the past framework and opens at the same time, another "world". It indicates, in any case, that it is certainly time for reinventing.

After a social growth model, we are witnessing a waltz of models. Within this specific context of merger, reorganization, consolidation, relocation, increasing scarcity of labor, are we in search of a lost or do we work to develop a new model ? Are we working to develop new action's intelligence?

Create favorable conditions for the emergence of resilience contexts contributes to making systems operating or functional. An organization or system is not resilient in itself, individuals have this ability. It is in the interest of the organization to work with people who build their personal resilience. To cope with difficult situations, Weick emphasizes the importance of giving meaning to what is experienced, to consider the construction of meaning as a theoretical framework. It is as if these elements contribute to the emergence of a new organizational reliability. Weick defines the organizational reliability as a reflection of the ability of individuals to organize and reorganize to anticipate and cope with unexpected and dangerous situations. He distinguishes the construction of meaning at the individual level of the collective sensemaking.(4)

\section{Resilience and crisis}

The recent acceptance of resilience is based on the idea that, after a disturbance, the system does not find in all cases a return to equilibrium. Resistance coexist with multiple adjustments and changes. Resilience is the property of a system, adapting its structure to change, however, retains the same path after a disturbance. The term resilience implies that the system maintains its structure and ensures its continuity, not maintaining an unchanging balance or returning to the same state as before the disturbance, but rather by incorporating some transformations. Using the crisis as a learning opportunity is drawing lessons arising from a previous experience in order to establish a new decision making process in a similar situation.

In connection with the work developed by the research laboratory CIRERO it became necessary to introduce a distinction in terms of resilience relative to the moment of a destabilizing event. CIRERO differentiate resilience 1 from resilience 2 . In summary, the resilience of type 1 expresses the person's ability to mobilize necessary energies to regain a state of equilibrium prior to the shock within acceptable limits. The time perspective is the present and the past. The resilience of type 2 expresses the person's ability to mobilize required energies to get through the crisis and find a new balance out in rupture with the previous situation. The time perspective is the present and the future.

Resilience is part of the time, it does not correspond to a state, but at a constant evolution. Indeed, resilience activates capabilities to develop interfaceable structures that can adapt as quickly and as easily as possible to all the organization into a shock situation. The resilience of type 1 and type 2 should not be treated as the process of homeostasis to make the necessary adjustments to maintain the balance. Indeed, resilience precedes the process of homeostasis or change. Resilience is the ability to release the required energy to initiate the process. In this sense, resilience is a necessary condition to 
the change process. Resilient feelings of effectiveness enables people to achieve extraordinary things through productive use of their skills facing difficult hurdles to overcome. Only the implementation of the conditions of resilience allows the organization to bounce favorably (resilience type 1) or exceed its origin (resilience type 2). Each new soubresaut of a traumatic event generates a new disturbance, an additional aggregate to the general collapse if nothing is implemented. The continuity of the organization is in danger.

Resilience is a resource to be deployed to stimulate rather autonomy, support, individual accountability and to bring the individual to control his professional and personal future. Addressing organizational resilience is to tackle the daily and the future of our organizations. It is indeed one of the levers to help people to bounce back, to facilitate organizational change, to cross turbulences and keep the course.

\section{Conclusions and perspectives}

The preceding decades we have been engaged in the development of security-based systems. Welfare systems safety and labor law are witnesses. Devices regulate various life events to the point of asserting the rights and forgetting duties. In the present context of mergers, restructurings, relocations, business closures and globalization put us up against the wall. Certainties are severely tested.

It is as though we are amputees resources to deal with the uncertainties of everyday life. It is as we are witnessing an atrophy of knowledge to face our future. Blinded by the events we are unable to establish a link between the past and the future. We reproduce on a recurring basis approaches based on the knowledge of the past while remaining within a limited world. We are still stuck in the swirl while a jump is to be made. The paradigm which has guided us has to be revisited.

How the decline the new paradigm in such context?

"We can get out of tough situations and even hopeless."

"Trauma can be repaired and be healed."

"Undergone death will not be accepted, we have to fight."

"Ensure that death drive be generator drive of life."

"Build the appropriate use of failures, difficulties."

"The life instinct which carries the forces of death is not feared."

"The war of life against death."

"The myth of life against all."

A new approach is to take a different perspective on the crisis, enlighten our minds otherwise give credit to new knowledge and take into account the conditions for the emergence of resilience processes and the deployment of associated skills.

Enable process based on the paradigm supplying resilience type 2 while maintaining active the resilience of type 1: the one and the other. Both are to be activate. It is as appropriate to combine knowledge and resilience processes of type 2 to find new equilibria. Deploy the resilience of type 2 that amounts to creating an opening for knowledge of a different kind. It acts as a activator.

\section{Biography}

Guy Koninckx

Bachelor of Science in Labor and Industrial Psychology, Guy Koninckx has an extensive experience in the Human Resources Management. This allows him to have a look at different organizational cultures. Systemics were a valuable approach to diagnose and manage complex situations. He joined GIROS and cofounded S \& O - Systems and Organizations. For many years he contributed to the drafting committee of HR Tribune - a journal devoted to human resources. Under Namastis, he provides advice and provides expertise to organizations faced with change management. He supports teams and individuals facing difficult situations, willing to change. Questioned by upheavals faced by 
organizations and individuals, he conducts studies and research focused on organizational resilience together with Gilles Teneau.

\section{References}

(1) C. Aschan-Leygonie, Maître de conférence à l'Université de Lyon 2. Partie 3 Connaissance et gouvernance territoriale, de nouvelles pistes pour accompagner le développement saharien, p 273.

(2)A. Piecq De la pensée systémique à la pratique de l'organisation - Le «giroscope »", Paris, Ed. l'Harmattan, 2011, p 138-139.

(3) B.Vidaillet, The Meaning of the action, Paris, Ed. Vuibert, 2003, p. 180.

(4) G. Koninckx ,G. Teneau, Résilience organisationnelle - Rebondir face aux turbulences, Bruxelles, Ed. De Boeck, 2010, p47-52

(5) G. Koninckx ,G. Teneau, op.cit., p 74 